\title{
The Impact of COVID-19 Pandemic on Rehabilitation in Singapore
}

\section{Dear Editor,}

The COVID-19 pandemic began in November 2019 and has affected over 62 million people worldwide with over 1.46 million deaths reported. ${ }^{1}$

The impact of the COVID-19 pandemic has been disruptive on rehabilitation systems worldwide. In this article, we describe the impact of COVID-19 to rehabilitation medicine practice in Singapore, and provide suggestions to improve the delivery of rehabilitation in future pandemics.

Inpatient rehabilitation bed capacity. Due to the urgent demand and rising need for inpatient management, many rehabilitation wards were converted to general medical wards or isolation wards to house COVID-19 patients. Community hospitals were converted to community isolation facilities or had a significant proportion of their existing beds re-designated to manage subacute COVID-19 patients. ${ }^{2}$ Therefore, there was a significant reduction in bed capacity for patients undergoing rehabilitation in both acute and community hospitals across Singapore.

Discharges from acute hospital inpatient rehabilitation units and from community hospitals to residential long-term community facilities and nursing homes were delayed due to insufficient beds, resources and patients' fear of infection. Similarly, hospitals in the US had a reduction in post-discharge options such as skilled nursing facilities, due to both bed and manpower shortages. ${ }^{3}$ These changes have particularly impacted the ability of both allied health professionals and rehabilitation physicians to adequately assess and manage patients with conditions that require require timely, multidisciplinary rehabilitation, such as stroke, spinal cord or traumatic brain injuries.

Length of rehabilitation stay. The growing pressure to free up beds for COVID-19 patients by expediting inpatient rehabilitation discharges, coupled with patients' perceived concerns of getting infected, led to a reduction in rehabilitation length of stay and early discharges prior to attainment of rehabilitation goals. Singh et al. reported an urgent emphasis to prepare inpatients for early discharge with appropriate post-discharge care arrangements rather than optimising inpatient functional recovery in rehabilitation units in the UK. ${ }^{4}$

Re-deployment of manpower. There were significant challenges in the daily provision of inpatient rehabilitation services because of the redeployment of rehabilitation physicians, nurses and allied health personnel to manage COVID-19 wards, general medicine wards and fever screening areas in emergency departments. This is similar in other countries. ${ }^{5}$

Inpatient rehabilitation services. Many inpatient rehabilitation services were ceased or modified to adhere to social distancing measures and reduce cross-infection. These include the suspension of home visits for group therapy strategies. Regular multidisciplinary meetings were conducted on virtual platforms such as Zoom (San Jose, CA, US) or Webex (Milpitas, CA, US). Hospitals in the UK reported higher thresholds for home visits, and therapy sessions limited to immediate bed space as part of COVID-19 infection control strategies. ${ }^{6}$

The closure of community care centres for the disabled, and the reduced inflow of foreign domestic helpers as caregivers for the newly disabled, have led to delays in transfer to community hospitals from acute hospitals. They have also caused delays in discharges to the home and community settings for both acute hospitals and community hospitals.

Outpatient-based acute hospital rehabilitation and rehabilitation medicine clinics. COVID-19 lockdown measures have affected outpatient rehabilitation services. Firstly, patients and their families were not keen to return to tertiary hospitals for follow-up clinic visits. Secondly, with the deployment of manpower to COVID-19 wards and screening, there were less rehabilitation medicine physicians supporting the core rehabilitation clinical services. Consequently, clinic sessions were either closed or limited to a few patients.

As many of the follow-up appointments with rehabilitation medicine physicians were postponed, the supply of medication was couriered to patients' homes. A small minority of patients returned to the clinic for assessment and interventions, when deemed necessary by the attending rehabilitation medicine physician. Some 
institutions have also adopted teleconsultation via audio or video calls. However, this is greatly limited by the inability of a physician to perform a comprehensive physical examination ${ }^{7}$ and certain interventions, such as intra-articular injections and application of orthoses.

Most outpatient rehabilitation services in acute hospitals were suspended during the COVID-19 lockdown period. There were tight criteria for patient selection in scheduling of face-to-face therapy sessions. Telerehabilitation was initiated to some extent, but its usage was limited as many patients had cognitive impairments and severe disability that required physical assistance, or they lacked a caregiver or access to the required digital platforms. As such, many patients were provided with a home exercise and rehabilitation plan prior to discharge to mitigate the impact of these restrictions.

Moving forward, planning for increasing rehabilitation needs amid threat of future pandemics.

Rehabilitation is an essential service. Patients with timesensitive conditions, such as stroke or spinal cord injury, require both inpatient and outpatient programmes to utilise the window of opportunity to reduce their disability. Hence maintaining a minimum level of hospital and community-based rehabilitation services in future pandemics would have significant economic and psychosocial impact on individuals and the society as a whole post-pandemic. We propose the following ways to improve Singapore's rehabilitation systems post COVID-19:

Triage by condition. The local rehabilitation community should develop a prioritised list of conditions in which rehabilitation has to continue in a pandemic. There should be a minimum number of inpatient rehabilitation beds in each hospital to receive acute major debilitating conditions such as stroke, spinal cord or traumatic brain injuries. Although outpatient rehabilitation is limited due to safe-distancing requirements, sufficient sessions to cater to discharged inpatients or existing communitydwelling persons at risk of significant functional decline should still be provided. Close collaborative work between rehabilitation clinicians and healthcare authorities will help facilitate these transitions successfully.

Triage by severity. There should be an understanding at both community and acute hospital settings that patients with mild to moderate disability or stable conditions can be followed up via phone/telerehabilitation/teleconsultation means, while those with severe disability or at risk of further functional decline must still come in person for outpatient therapy or rehabilitation medicine clinic sessions. These same principles should also guide homebased rehabilitation service patient prioritisation.
In-reach and follow-through by rehabilitation physicians. Inpatient pathways and in-reach services should be developed and led by rehabilitation physicians for early, multidomain rehabilitation assessments and functional prognostication, especially in patients with stroke, spinal cord or traumatic brain injuries, and those at risk of post-intensive care syndrome. This ensures that early, coordinated, multidisciplinary rehabilitation occurs throughout the patient's inpatient journey for optimal rehabilitation at all times.

Inpatient rehabilitation facility planning. Designating which community hospitals and acute hospital inpatient rehabilitation units should receive pandemic or nonpandemic patients will allow for optimal planning of bed capacity and healthcare manpower utilisation in a pandemic. For example, pre-designating certain community hospitals as community isolation facilities beforehand will allow senior management to allocate a number of their medical, nursing and rehabilitation staff to attend to patients afflicted by the pandemic with or without rehabilitation needs, while the remaining staff can be deployed to other non-pandemic community hospitals to serve patients with functional loss not due to the particular pandemic disease. This reduces the chance of cross-infection in the same facility and possibly allow for a better efficiency for acute to community hospital transfers, ultimately alleviating the bed capacity pressure on acute hospitals in pandemic times.

Infection control considerations in inpatient and outpatient rehabilitation settings. Rehabilitation is unique in that it involves shared equipment in dedicated spaces and group therapy. There should be clear protocols in place in a pandemic about the required personal protective equipment, cleaning of rehabilitation equipment used, as well as limits to the number of patients allowed in a group in both in- and outpatient settings. This reduces the risk of disease transmission, allows for optimal deployment of rehabilitation manpower, potentially reducing costs through bulk buying agreements in the purchase of cleaning materials and services at the hospital level.

We believe that rehabilitation is an essential service during pandemics. Rehabilitation medicine physicians continue to be consulted to review patients with stroke, spinal cord injury, lower limb amputation, in both local acute and community hospitals because of their broad-based training, throughout the lockdown period.

Rehabilitation medicine has an important role in managing new-onset disability from COVID-19 or otherwise, as well as preventing future complications. In addition to providing rehabilitation services, rehabilitation 
physicians in Singapore are well-equipped to provide acute and general medical care where required. There is a need to streamline rehabilitation care pathways during a pandemic so that patients continue to receive rehabilitation to optimise their recovery.

In the lead author's institution, rehabilitation physicians are involved in consultations of COVID-19 patients as part of an interdisciplinary early rehabilitation initiative, which extends from the intensive care unit (ICU) to the general isolation ward, and to outpatient follow-up. Areas of assessment and management that the rehabilitation physician focuses on include assessment and management of ICU-associated weaknesses, reduced pulmonary endurance post COVID-19 pneumonia, ${ }^{8} \mathrm{ICU}$-associated delirium and post-discharge follow-up, community re-integration and return to work. All these are done as part of a holistic, interdisciplinary effort to ensure that COVID-19 ICU patients not only survive, but survive well.

\section{REFERENCES}

1. World Health Organization. WHO coronavirus disease (COVID-19) dashboard. Available at: https://covid19.who.int/ gclid=CjwKCAjwmrn5BRB2EiwAZgL9ok3G0KTVBU_c9x9z yatfP2Vi1DdV53zbbmQ9Oanxc-9VavgOwv MBoC0RsQAvD BwE208/8/2020. Accessed on 2 December 2020.

2. Iau J. Bright Vision Hospital transfers all patients to make room for stable Covid-19 cases. Available at: https://www.straitstimes.com/ singapore/health/bright-vision-hospital-transfers-all-patients-to-makeroom-for-stable-covid-19. Accessed on 24 May 2020.

3. Bettger J, Thoumi A, Marquevich V, et al. COVID-19: Maintaining essential rehabilitation services across the care continuum. BMJ Glob Health 2020;5:e02670.

4. Singh R, Burn J, Sivan M. A letter in response to the SIMFER document on the impact of COVID-19 on Italian rehabilitation services and activities. Eur J Phys Rehabil Med 2020;56:368-369.
5. Stein J, Visco C, Barbuto S. Rehabilitation medicine response to the COVID-19 pandemic. Am J Phys Med Rehabil 2020;99:573-9.

6. Mamo J, Feroz B, Mahmood S. Covid-19: Protecting patients in hospital for neurorehabilitation and their therapists. BMJ 2020;369:m1630.

7. Turolla A, Rossettini G, Viceconti A, et al. Musculoskeletal physical therapy during the COVID-19 pandemic: is telerehabilitation the answer? Phys Ther 2020;100:1260-4.

8. Tay SS, Neo EJR, Tan MMJ, et al. Post-critical care COVID-19 patient benefits from a robotic patient-guided suspension system for pulmonary rehabilitation. Ann Acad Med Singap 2020;49:401-4.

Dominic EH Chen, ${ }^{1}$ MBBS (S'pore), MRCP (UK), MMed (Int Med, S'pore),

Elvina KW Tay, ${ }^{2}$ MBBS (S'pore), MRCP (UK), FAMS ( $S^{\prime}$ pore),

Pei Ling Tan, ${ }^{3}$ MD (S'pore), MRCP (UK), FAMS,

Peijing $\underline{\mathrm{Su}},{ }^{4}{ }_{M B B S}$ (London), MRCP (UK),

Moses MH Koh, ${ }^{5}$ MBBS (Adelaide), MRCP (UK),

Poo Lee Ong, $\left.{ }^{6}{ }_{M B B S}(U K M), M R C P(U K)\right)$,

Effie Chew, ${ }^{4}$ MBBS (Melbourne), MRCP (UK), FAMS (S'pore),

Yee Sien $\mathrm{Ng},{ }^{5}$ MBBS (S'pore), MRCP (UK), FAMS (S'pore)

\footnotetext{
${ }^{1}$ Department of General Medicine, Rehabilitation Medicine Service, Sengkang General Hospital, Singapore

${ }^{2}$ Department of Post-Acute and Continuing Care, Jurong Community Hospital, Singapore

${ }^{3}$ Department of Rehabilitation Medicine, Changi General Hospital, Singapore

${ }^{4}$ Department of Rehabilitation Medicine, National University Hospital, Singapore

${ }^{5}$ Department of Rehabilitation Medicine, Singapore General Hospital, Singapore

${ }^{6}$ Department of Rehabilitation Medicine, Tan Tock Seng Hospital, Singapore
}

Address for Correspondence: Dr Dominic EH Chen, Department of General Medicine, Rehabilitation Medicine Service, Sengkang General Hospital, 110 Sengkang East Way, Singapore 544886.

Email: dominic.chen.enhan@singhealth.com.sg 